\title{
Clinical Features and Prognosis of Sudden Sensorineural Hearing Loss Secondary to Intralabyrinthine Hemorrhage
}

\author{
Jae Woo Lee, Yoon Ah Park, Sang Man Park, Tae Hoon Kong, Sang Yoo Park, \\ Jeong Pyo Bong, Dong-Joon Park, and Young Joon Seo \\ Department of Otorhinolaryngology, Yonsei University Wonju College of Medicine, Wonju, Korea
}

Received October 26, 2015

Revised November 18, 2015

Accepted December 23, 2015

Address for correspondence

Young Joon Seo, MD, PhD

Department of Otorhinolayngology,

Yonsei University Wonju

College of Medicine,

20 Ilsan-ro, Wonju 26426, Korea

Tel +82-33-741-0644

Fax $+82-33-732-8287$

E-mail okas2000@hanmail.net
Background and Objectives: A number of etiologies of idiopathic sudden sensorineural hearing loss (ISSNHL) have been proposed, including viral infection, vascular disturbance, and immune-mediated mechanisms. Intralabyrinthine hemorrhage (ILH) as a cause of SSNHL is extremely rare, and there have been no studies defining the characteristics of hearing impairment and prognosis in patients with ISSNHL due to ILH. This study aimed to investigate the difference in impaired hearing patterns and prognosis for hearing recovery between patients with ISSNHL due to ILH confirmed by magnetic resonance imaging (MRI) and sex- and age-matched patients with ISSNHL due to causes other than ILH. Subjects and Methods: We compared the results of audiometry and MRI in 12 patients who had ILH on MRI (hemorrhage group) and in 23 sex- and age-matched controls without abnormal findings related to their hearing loss on MRI (non-hemorrhage group). Initial hearing impairment, progression, and recovery of hearing loss were compared between the two groups. Results: A majority of patients (92\%) in the hemorrhage group complained of dizziness. Initial hearing impairment was more frequent in the hemorrhage group than in the non-hemorrhage group ( $94.09 \pm 35.9$ vs. $66.66 \pm 30.1, p$-value $=0.036)$. The final recovery threshold in the hemorrhage group was worse $(78.19 \pm 46.26$ vs. $37.17 \pm 31.96, p$-value $=0.014)$ than that in the non-hemorrhage group. In the hemorrhage group, hearing recovery seemed to occur less often at high frequencies $(2,000,4,000$, and $8,000 \mathrm{~Hz})$ than at low frequencies $(250$, 500 , and $1,000 \mathrm{~Hz}$ ). Conclusions: The presence of ILH was associated with poor hearing prognosis and the occurrence of vertigo. The abrupt onset of hearing loss associated with vertigo and the presence of hyperresonance on fat-suppressed T1-weighted MRI images of labyrinthic fluid strongly suggests acute intralabyrinthine hemorrhage, and is predictive of considerable hearing impairment and poor prognosis. J J Audiol Otol 2016;20(1):31-35

KEY WORDS: Sudden sensorineural hearing loss · Intralabyrinthine hemorrhage · Magnetic resonance imaging $\cdot$ Prognosis.

\section{Introduction}

A common definition of idiopathic sudden sensorineural hearing loss (ISSNHL) is a loss of greater than $30 \mathrm{~dB}$ in three contiguous frequencies in less than three days [1]. A number of ISSNHL etiologies have been proposed, including viral infection, vascular disturbance, and immune-mediated mech-

This is an Open Access article distributed under the terms of the Creative Commons Attribution Non-Commercial License (http://creativecommons. org/licenses/by-nc/3.0/) which permits unrestricted non-commercial use, distribution, and reproduction in any medium, provided the original work is properly cited. anisms. However, there is no conclusive evidence for any particular hypothesis [2-4].

An audiogram determines whether an acute hearing loss is sensorineural, conductive, or mixed. When hearing loss is identified, further evaluation should be carried out with computed tomography (CT), which is more sensitive in conductive hearing loss, and magnetic resonance imaging (MRI), which is more sensitive in sensorineural hearing loss. Intralabyrinthine hemorrhage (ILH) in patients with ISSNHL can be clearly detected on MRI. ILH as a cause of SSNHL is extremely rare, and there have been few case reports in the liter- 
ature [5-8].

The characteristics of hearing impairment and prognosis in patients with ISSNHL secondary to ILH are also unclear. In this study, by comparing patients with ISSNHL and ILH visualized on MRI to sex- and age-matched patients with ISSNHL and no ILH, we were able to distinguish hearing impairment patterns and evaluate prognosis in terms of hearing recovery in patients with ILH.

\section{Subjects and Methods}

\section{Subjects}

We retrospectively reviewed the records of 1046 consecutive patients with ISSNHL treated in our department from 2009 to 2013. We defined ISSNHL as acute unilateral deafness (generally within three days) defined as hearing loss of more than $30 \mathrm{~dB}$ at three consecutive frequencies. We ultimately included 12 patients (hemorrhage group) who had ILH on MRI diagnosed by one neuroradiologist and another trained otolaryngologist. The control group (non-hemorrhage group) included 23 sex- and age-matched subjects who had no abnormal findings related to their hearing loss on MRI. None of the study participants had a neurological abnormality, history of myocardial infarction or head trauma. All patients were treated with prednisone $1 \mathrm{mg} / \mathrm{kg}$ per day for 7 days with progressive dose reduction. None of the patients was treated with intratympanic steroid injection.

\section{MRI acquisition and processing}

All MRIs were performed with a 3T MR unit (Magnetom Trio; Siemens, Erlangen, Germany) using a receive-only, 12-channel, phased-array coil. The scan parameters for 3DFLAIR were as follows: repetition time, 9,000 ms; effective echo time, $128 \mathrm{~ms}$; inversion time, 2,500 ms; flip angle, 1,808 (constant); turbo spin-echo refocusing echo train length, 23; matrix size, $384 \times 384$; and 12 axial 2-mm-thick sections covering the labyrinth with a $16-\mathrm{cm}$ square field-of-view acquired using the generalized autocalibrating partially parallel acquisition parallel imaging technique with an acceleration factor of 2. We defined ILH on MRI as high signal on unenhanced T1-weighted (T1W) sequence and on T2-weighted (T2W) sequence (Fig. 1) [9,10]. We considered only a unilateral hyperintense lesion on $\mathrm{T} 1 \mathrm{~W}$ imaging when compared with the surrounding labyrinth and on T2W imaging with a high cerebrospinal fluid intensity.

\section{Auditory evaluation}

Pure-tone thresholds were obtained for air conduction at $250 \mathrm{~Hz}, 500 \mathrm{~Hz}, 1 \mathrm{kHz}, 2 \mathrm{kHz}, 4 \mathrm{kHz}$, and $8 \mathrm{kHz}$ and for bone conduction at $250 \mathrm{~Hz}, 500 \mathrm{~Hz}, 1 \mathrm{kHz}, 2 \mathrm{kHz}$, and 4 $\mathrm{kHz}$. Audiologic data were reported based on the methods recommended by the Hearing Committee of the American Academy of Otolaryngology Head and Neck Surgery. Patients were evaluated according to the recovery that was seen after a maximum of two months of follow-up. Pure tone audiometry was performed with patient responses to the treatment classified according to Siegel's $[11,12]$ criteria. Then, we defined recovered (complete+partial+slight) and "unrecovered (no improvement)". "Recovery time" means the time at which the patient's hearing first recovered according to Siegel's criteria.

\section{Statistical analyses}

Continuous variables were summarized as mean \pm standard deviation and comparisons between continuous variables were performed using Student's t-test. Categorical variables were summarized as percentages of the group total and comparisons between groups were performed using either Fisher's exact test or the chi-square test where appropriate. All tests used a $p$-value of 0.05 as the threshold for significance,
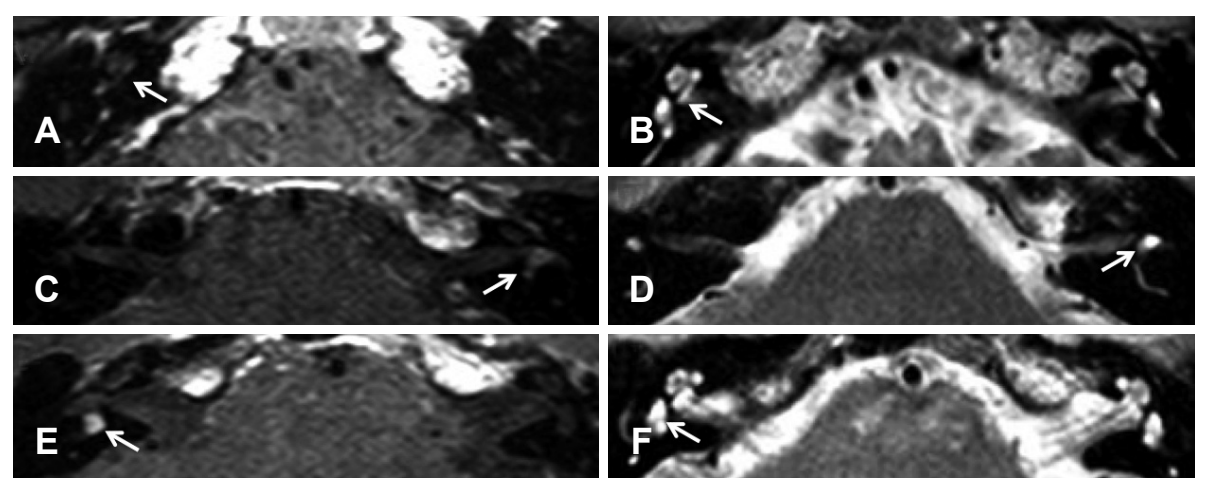

Fig. 1. Magnetic resonance imaging of the temporal bones; axial slices. A: T1-weighted image before intravenous contrast showing a hyperintense signal in the basal turn of the right cochlea (arrow). B: T2-weighted image demonstrating a high signal (arrow) in the same location. T1-weighted image (C) and T2 weighted image (D) showing a hyperintense signal in the left anterior semicircular canal (arrow). T1-weighted image (E) and T2 weighted image (F) showing a hyperintense signal in the right vestibule (arrow). 
and all statistical analyses were performed using SPSS Software (PASW for Windows, Rel. 18.0.0. 2009; SPSS Inc., Chicago, IL, USA).

\section{Results}

Patient demographics are summarized in Table 1. Lesion laterality was $7 / 12(58 \%)$ in the right ear and 5/12 (42\%) in the left ear, and 11/12 (92\%) patients had a cochlear hemorrhage. Hemorrhage of the vestibule and semicircular canal (SCC) were suspected in $42 \%$ and $50 \%$, respectively. The majority of patients (92\%) complained of dizziness, but the frequency of hemorrhagic lesions on vestibular structures was not correlated with this symptom.

There were no significant differences in age, gender, BMI, affected ear and hematologic tests between the two groups (Table 2) ( $p$-values $>0.05$ ). However, there were significant differences in the prevalence of dizziness, initial hearing level, and recovered hearing level. The initial hearing impairment was higher in the hemorrhage group than in the non-hemorrhage group $(94.09 \pm 35.9$ vs. $66.66 \pm 30.1, p$-value $=0.036)$. The final recovery threshold in the hemorrhage group was worse $(78.19 \pm 46.26$ vs. $37.17 \pm 31.96, p$-value $=0.014)$, but the final recovery time did not differ significantly between the groups ( $p$-value $=0.575$ ).

The hearing patterns are shown in Fig. 2. Hearing impair-

Table 1. Demographics of patients with intralabyrint

\begin{tabular}{|c|c|c|c|c|c|c|c|c|c|c|}
\hline \multirow{2}{*}{ Patient No. } & \multirow{2}{*}{ Sex } & \multirow{2}{*}{ Age } & \multirow{2}{*}{ Lesion side } & \multirow{2}{*}{ Dizziness } & \multirow{2}{*}{$\begin{array}{l}\text { Initial thresholds } \\
\text { of PTA }(d B)\end{array}$} & \multirow{2}{*}{$\begin{array}{c}\text { Final thresholds } \\
\text { of PTA }(d B)\end{array}$} & \multirow{2}{*}{$\begin{array}{c}\text { Recovery } \\
\text { duration (days) }\end{array}$} & \multicolumn{3}{|c|}{ MRI } \\
\hline & & & & & & & & Cochlea & Vestibule & SCC \\
\hline 1 & $F$ & 55 & Right & Yes & 120 & 105 & 7 & - & - & + \\
\hline 2 & $\mathrm{~F}$ & 55 & Left & Yes & 120 & 120 & 43 & + & - & - \\
\hline 3 & $\mathrm{~F}$ & 22 & Right & Yes & 48.75 & 30 & 21 & + & - & - \\
\hline 4 & M & 15 & Right & Yes & 95 & 26.25 & 44 & + & + & + \\
\hline 5 & M & 37 & Left & Yes & 110 & 98.75 & 30 & + & + & + \\
\hline 6 & M & 71 & Left & Yes & 117.5 & 112.5 & 42 & + & + & + \\
\hline 7 & M & 45 & Right & No & 93.75 & 120 & 84 & + & + & + \\
\hline 8 & $F$ & 52 & Left & Yes & 120 & 113.75 & 72 & + & - & - \\
\hline 9 & $\mathrm{~F}$ & 63 & Left & Yes & 120 & 108.75 & 50 & + & + & + \\
\hline 10 & $\mathrm{~F}$ & 45 & Right & Yes & 20 & 10 & 35 & + & - & - \\
\hline 11 & M & 52 & Right & Yes & 117.5 & 83.75 & 18 & + & - & - \\
\hline 12 & $\mathrm{~F}$ & 19 & Right & Yes & 43.75 & 6.25 & 21 & + & - & - \\
\hline
\end{tabular}

M: man, F: woman, PTA: pure tone audiometry, SCC: semicircular canal, MRI: magnetic resonance imaging

Table 2. Comparison of patient demographics between the hemorrhage group and the non-hemorrhage group

\begin{tabular}{lccc}
\hline Variables & Hemorrhage $(\mathrm{n}=12)$ & Non-hemorrhage $(\mathrm{n}=23)$ & $p$-value \\
\hline Age (years) & $44.25 \pm 17.73$ & $45.34 \pm 16.74$ & 0.861 \\
Gender, male/female (number) & $5 / 7$ & $9 / 14$ & 0.583 \\
BMI $\left(\mathrm{kg} / \mathrm{m}^{2}\right)$ & $25.29 \pm 4.98$ & $23.35 \pm 2.77$ & 0.250 \\
Affected ear (R/L) & $7 / 5$ & $10 / 13$ & 0.316 \\
Dizziness & $11(92 \%)$ & $6(26 \%)$ & $<0.001^{*}$ \\
Initial hearing level (dB) & $94.09 \pm 35.9$ & $66.66 \pm 30.1$ & $0.036^{*}$ \\
Final recovery hearing level (dB) & $78.19 \pm 46.26$ & $37.17 \pm 31.96$ & $0.014^{*}$ \\
Final recovery time (days) & $38.92 \pm 22.35$ & $34.00 \pm 27.71$ & 0.575 \\
Hemoglobin $\left(10^{3} / \mathrm{u}\right)$ & $14.69 \pm 1.35$ & $14.23 \pm 1.31$ & 0.342 \\
WBC $\left(10^{3} / \mathrm{U}\right)$ & $10.02 \pm 6.07$ & $9.54 \pm 3.92$ & 0.807 \\
Platelet $\left(10^{3} / \mathrm{u}\right)$ & $272.75 \pm 84.16$ & $286.39 \pm 50.74$ & 0.614 \\
PT (sec) & $10.86 \pm 0.66$ & $10.86 \pm 0.92$ & 0.769 \\
PT (INR) & $0.98 \pm 0.06$ & $0.98 \pm 0.08$ & 0.744 \\
PTT (sec) & $27.26 \pm 4.75$ & $29.53 \pm 2.84$ & 0.164
\end{tabular}

Data with a normal distribution is expressed as the mean \pm standard deviation and as a number with percentage for categorical variables. *a p-value<0.05 value was set as the significance level, and significant differences between groups are shown in symbol. BMI: Body Mass Index, R/L: right/left ears, WBC: white blood cell, PT: prothrombin time, INR: international normalized ratio, PTT: partial thromboplastin time, sec: seconds 


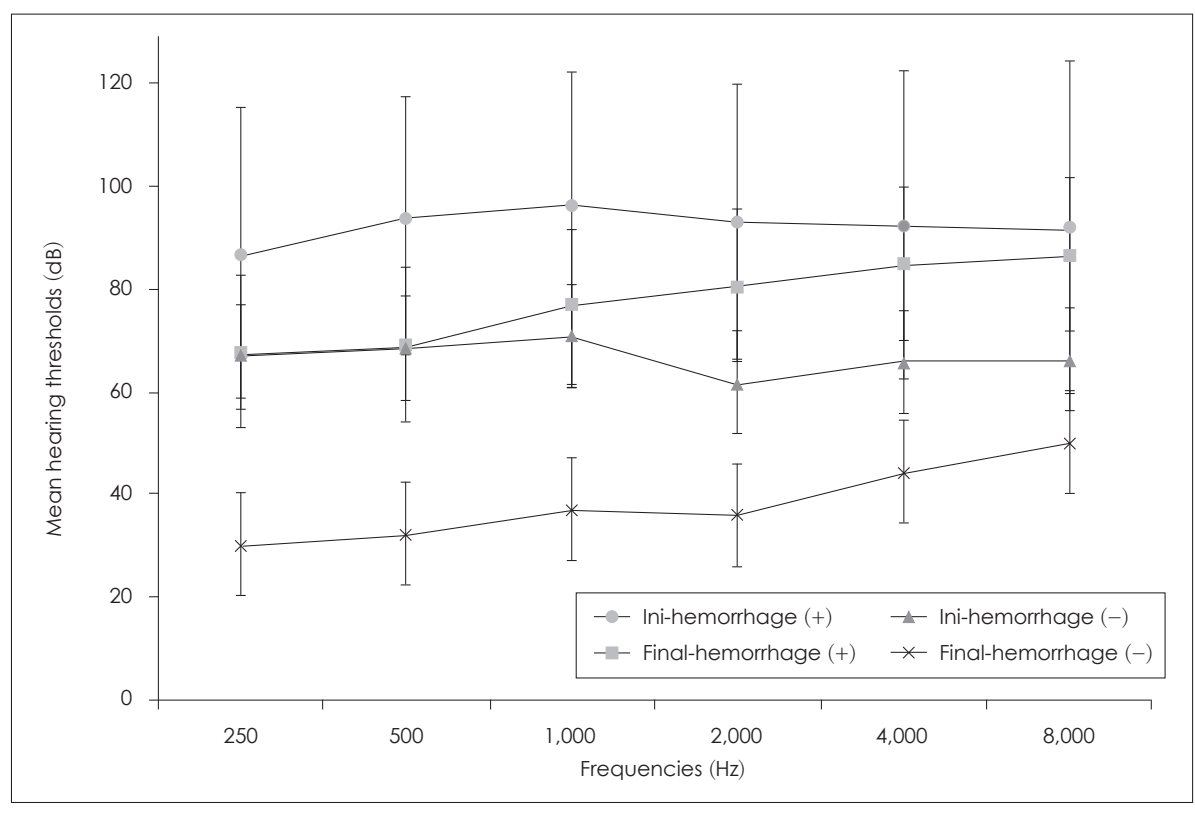

Fig. 2. The results of pure tone audiometry before and after treatment between the hemorrhage and nonhemorrhage groups. ment in the group with ILH was more profound than that in the group without ILH at every frequency. In the hemorrhage group, hearing recovery was less pronounced at high frequencies $(2,000,4,000$, and $8,000 \mathrm{~Hz})$ than at low frequencies $(250,500$, and $1,000 \mathrm{~Hz})$.

\section{Discussion}

ISSNHL secondary to ILH is a rare diagnosis best made on MRI. It is typically unilateral, and may be partial or complete, and sudden or rapidly progressive [6]. However, no study has described the characteristics of hearing impairment and prognosis in patients with ISSNHL due to ILH [7]. In our study, we determined the quantitative difference in hearing impairment between the hemorrhage and non-hemorrhage groups (94.09 dB vs. $66.66 \mathrm{~dB}$ ). Furthermore the hearing recovery over two months was significantly worse in the hemorrhage group ( $p$-value $=0.014$ ). The symptom of dizziness was more frequent $(92 \%)$ in patients with ILH. The patients with ISSNHL and ILH seemed to suffer from worse hearing impairment and prognosis than patients with ISSNHL due to other causes.

Causes of SSHL may be infectious (viral or bacterial), vascular, immune mediated (within the inner ear or systemically), or resulting from neurological disease (migraine, multiple sclerosis), neoplasms or ototoxicity. Potential vascular etiologies of ISSNHL include hemorrhagic and obstructive. ILH has been described in patients with aplastic anemia, sickle-cell disease, leukemia, secondary to cranial trauma, and following surgery for the treatment of vestibular schwannomas $[10,13-15]$. However, only three $(25 \%)$ of our patients had histories of anticoagulation therapy, and there were no suspicious causes of ILH in the remaining patients.

MRI is an established diagnostic modality in the evaluation of patients with ISSNHL. Although a distinct pathology can only be identified in $10 \%$ of patients, the differential diagnosis includes infection, trauma, neoplastic, immunologic, toxic, vascular compromise, and neurologic and metabolic causes [6]. In MRI, T1 hyperintense signals can in theory result from fat, protein, or blood [8]. Normally, perilymph and endolymph are isointense to cerebrospinal fluid on all MRI sequences. Fat, proteinaceous materials and methemoglobinemia will all cause hyperintensity on T1W imaging, but fat can be distinguished with suppression. ILH is characterized on MRI by high signal intensity on T1W imaging yet will not enhance with gadolinium. Plain T2W images are not helpful because signal intensity can be variable. Labyrinthine schwannomas differ from ILH on MRI by marked enhancement following the administration of intravenous contrast medium (gadolinium). We defined ILH on MRI as high signal both on unenhanced $\mathrm{T} 1 \mathrm{~W}$ sequence and on $\mathrm{T} 2 \mathrm{~W}$ sequence identified in consensus by one neuroradiologist and a trained otolaryngologist. We identified cochlear (92\%), vestibular (42\%) and SCC (50\%) ILH.

Yoshida, et al. [16] felt that high signals in the cochlea on pre-contrast 3D-FLAIR MRI were related to a poor hearing prognosis in patients with sudden hearing loss. Berrettini, et al. [17], however, found no such hearing correlation, but did associate vertigo with high signal intensity. In our study evaluating patients with sudden hearing impairment due to ILH, the presence of ILH was associated with poor hearing 
prognosis and the presence of vertigo when compared with sex- and age-matched controls without ILH on MRI. To our knowledge, this is the first study to describe the characteristics and prognosis of ILH.

In conclusion, this study aimed to investigate the difference in hearing impairment patterns and hearing recovery prognosis between patients with ISSNHL and ILH on MRI to sex- and age-matched patients with ISSNHL but not ILH. We determined that the initial hearing impairment was greater and the final recovered hearing was worse in the hemorrhage group than in the non-hemorrhage group. The abrupt onset of hearing loss associated with vertigo and the presence of a hyperintense signal on fat-suppressed T1W MRI images of labyrinthic fluid strongly suggests acute intralabyrinthine hemorrhage, which is associated with considerable hearing impairment and a poor prognosis.

\section{Acknowledgments}

This research was supported by a grant of the Korea Health Technology R\&D Project through the Korea Health Industry Development Institute (KHIDI), funded by the Ministry of Health \& Welfare, Republic of Korea (grant number: HI15C2237).

\section{Conflicts of interest}

The authors have no financial conflicts of interest.

\section{REFERENCES}

1) Hughes GB, Freedman MA, Haberkamp TJ, Guay ME. Sudden sensorineural hearing loss. Otolaryngol Clin North Am 1996;29: 393-405.

2) Merchant SN, Durand ML, Adams JC. Sudden deafness: is it viral? ORL J Otorhinolaryngol Relat Spec 2008;70:52-60; discussion 602.

3) Masuda M, Kanzaki S, Minami S, Kikuchi J, Kanzaki J, Sato H, et al. Correlations of inflammatory biomarkers with the onset and prognosis of idiopathic sudden sensorineural hearing loss. Otol Neurotol 2012;33:1142-50.

4) Ryan AF, Harris JP, Keithley EM. Immune-mediated hearing loss: basic mechanisms and options for therapy. Acta Otolaryngol Suppl 2002;(548):38-43.

5) Weissman JL. Hearing loss. Radiology 1996;199:593-611.

6) Rosado WM Jr, Palacios E. Sudden onset of sensorineural hearing loss secondary to intralabyrinthine hemorrhage: MRI findings. Ear Nose Throat J 2008;87:130-1.

7) Pézier T, Baráth K, Hegemann S. Partial recovery of audiological, vestibular, and radiological findings following spontaneous intralabyrinthine haemorrhage. Case Rep Otolaryngol 2013;2013: 941530.

8) Cervantes SS, Barrs DM. Sudden sensorineural hearing loss associated with intralabyrinthine hemorrhage. Otol Neurotol 2015;36: e134-5.

9) Palacios E, Valvassori G. Hemorrhagic labyrinthitis. Ear Nose Throat J 2000;79:80.

10) Weissman JL, Curtin HD, Hirsch BE, Hirsch WL Jr. High signal from the otic labyrinth on unenhanced magnetic resonance imaging. AJNR Am J Neuroradiol 1992;13:1183-7.

11) Siegel LG. The treatment of idiopathic sudden sensorineural hearing loss. Otolaryngol Clin North Am 1975;8:467-73.

12) Wilson WR, Byl FM, Laird N. The efficacy of steroids in the treatment of idiopathic sudden hearing loss. A double-blind clinical study. Arch Otolaryngol 1980;106:772-6.

13) Ogawa K, Kanzaki J. Aplastic anemia and sudden sensorineural hearing loss. Acta Otolaryngol Suppl 1994;514:85-8.

14) Whitehead RE, MacDonald CB, Melhem ER, McMahon L. Spontaneous labyrinthine hemorrhage in sickle cell disease. AJNR Am J Neuroradiol 1998;19:1437-40.

15) Sando I, Egami T. Inner ear hemorrhage and endolymphatic hydrops in a leukemic patient with sudden hearing loss. Ann Otol Rhinol Laryngol 1977;86(4 Pt 1):518-24.

16) Yoshida $T$, Sugiura $M$, Naganawa $S$, Teranishi $M$, Nakata $S$, Nakashima T. Three-dimensional fluid-attenuated inversion recovery magnetic resonance imaging findings and prognosis in sudden sensorineural hearing loss. Laryngoscope 2008;118:1433-7.

17) Berrettini S, Seccia V, Fortunato S, Forli F, Bruschini L, Piaggi P, et al. Analysis of the 3-dimensional fluid-attenuated inversion-recovery (3D-FLAIR) sequence in idiopathic sudden sensorineural hearing loss. JAMA Otolaryngol Head Neck Surg 2013;139:456-64. 